\title{
PENSAR EL ACONTECIMIENTO. UNA APROXIMACIÓN A LA PROPUESTA TRAUMÁTICA DE SLAVOJ ŽIŽEK
}

\section{THINKING ABOUT THE EVENT. AN APPROACH TO SLAVOJ ŽIŽEK'S TRAUMATIC PROPOSAL}

\author{
Esteban Andrés Sánchez Quintana \\ Universidad Pedagógica y Tecnológica de Colombia \\ estebandresanchez2@gmail.com
}

Recibido: 20 de abril de 2020 / Aceptado: 28 de abril 2020 / Publicado: 30 de junio de 2020

\section{Resumen}

El presente texto tiene como propósito abordar la categoría conceptual acontecimiento, siguiendo la perspectiva de Slavoj Žižek, quien sostiene que éste es un suceso traumático, porque en él, se hace patente el poder de lo real que condiciona la existencia y la realidad del ser humano. Para desarrollar el planteamiento anterior, se propone la siguiente estructura: para empezar, se busca definir, redefinir y enmarcar la categoría conceptual, luego, se pretende reflexionar cómo sucede este fenómeno en relación con la psiquis, en donde lo real es mediado por lo simbólico y lo imaginario. Finalmente, se busca pensar con el filósofo esloveno, qué se entiende por la condición traumática del acontecimiento.

Palabras clave: acontecimiento, trauma, real.

\begin{abstract}
The purpose of this article is to address some aspects of the conceptual category "event", following Slavoj Žižek's perspective. The structure of the text is as follows: To begin, the aim is to define, redefine and frame the concept. Next, an attempt will be made to account for how this phenomenon occurs in relation to the human psyche. Finally, think with the Slovenian philosopher, who is understood by the "traumatic" condition of the event.
\end{abstract}

Key words: event, trauma, real. 


\section{Definir, redefinir, enmarcar}

"Enigma que nace de un puro surgimiento". (Hölderlin, «El Rhin»)

Qué es un acontecimiento? Sin duda, la presente pregunta remite a múltiples posibilidades. Slavoj Žižek (2014, p. 15) resalta que, evidentemente, el concepto es una multiplicidad, puesto que lo reconoce como: "una noción amfibia con más de cincuenta tonos de gris. Un «acontecimiento» puede hacer referencia a un desastre natural devastador o al escándalo más reciente provocado por una celebridad, al triunfo del pueblo o a un cambio político despiadado, a la intensa experiencia de una obra de arte o a una decisión íntima”.

Así, el concepto podría hacer referencia a la forma de cortar el rumbo normal del entorno, pues, sin lugar a dudas, se relaciona con la manera en como se cambia el pensamiento, el contexto, el lenguaje, la ciencia, el arte o la cultura. En otras palabras, el acontecimiento sería la ruptura de la realidad a partir de un cúmulo de hechos deliberados, la aparición de circunstancias accidentales o el desocultamiento de un suceso misterioso.

De este modo, el fenómeno se puede relacionar con un descubrimiento científico, el planteamiento de un nuevo paradigma filosófico, el develamiento que hace manifiesta una obra de arte, una catástrofe natural o una revolución, es decir, es algo que marca un antes y un después. Por ello, un acontecimiento, visto de esta manera, podría ser la caída de las Torres Gemelas el 11 de septiembre de 2001 o la final de la Copa Mundo de la FIFA, como también podría ser la elección de un presidente en un país, el hallazgo de un manuscrito perdido de Aristóteles ${ }^{1}$, una pandemia de tintes apocalípticos o el estreno de una nueva película en Hollywood que rompa sus recaudos en taquilla.

Lo anterior permite inferir que hay diversos tipos de acontecimientos. Por ejemplo, existen históricos, sociales, naturales, políticos, deportivos,

1 Siguiendo este ejemplo se puede pensar, qué pasaría si el manuscrito de la comedia (de la Poética) de Aristóteles fuese descubierto en los tiempos de Edad Media. A nuestro parecer, eso sería un acontecimiento que hubiese cambiado el mundo y la relación dominante con Dios. Frente a lo enunciado recordamos a Umberto Eco en El nombre de la rosa (2013, p. 116) respecto al asunto de la relación entre la comedia y la verdad en la Poética de Aristóteles. "Ya lo oísteis ayer. Jorge señaló que no es lícito adornar con imágenes risibles los libros que contienen la verdad. Venancio observó que el propio Aristóteles había hablado de los chistes y de los juegos de palabras como instrumentos para descubrir mejor la verdad, y que, por tanto, la risa no debía de ser algo malo si podía convertirse en vehículo de la verdad. Jorge señaló que, por lo que recordaba, Aristóteles había hablado de esas cosas en el libro de la Poética y refiriéndose a las metáforas". 
científicos y artísticos. Sin embargo, no todos tendrían la misma importancia. Algunos serían de carácter general para la humanidad y otros obedecerían a particularidades de contextos o campos específicos del conocimiento. Ahora bien, se puede sostener que lo que tienen en común estos sucesos, de importancia generalizada o no, es que interrumpen el desarrollo normal de la vida y cambian la forma en como nos relacionamos con ella. No es de extrañar, por ello, que Raquel Vicedo (2014), traductora del libro referido, asegure que:

...en palabras del propio Žižek un acontecimiento «es algo traumático, perturbador, que parece suceder de repente y que irrumpe el curso normal de las cosas; algo que surge aparentemente de la nada, sin causas discernibles, una apariencia que tiene como base nada sólido», es por consiguiente, «el efecto que parece exceder sus causas». Un acontecimiento no solo reconfigura el presente y habilita un futuro impensable sin él, sino que redimensiona y articula el pasado que lo precede para que este pueda abrazarlo, encajarlo, explicarlo.

Se asume así al fenómeno como un suceso de una anormalidad extraordinaria. A partir de ahí, Žižek hace un llamado de atención sobre la discusión central del concepto, a saber: "en un primer enfoque, un acontecimiento es por consiguiente el efecto que parece exceder sus causas y el espacio de un acontecimiento es el que abre por el hueco que separa un efecto de sus causas" (2014, p. 17). Situación que se evidencia en una discusión entre lo trascendental y lo óntico, dicho en otras palabras, entre la filosofía y la ciencia.

Sin embargo, para este filósofo, la discusión tiene un punto de encuentro, esto es, que el acontecimiento no es un simple cambio, sino el suceso de redefinición o destrucción del planteamiento de la realidad. Por ello, a su entender:

...[independientemente de sí] ambos enfoques culminan en una determinada noción de Acontecimiento ${ }^{2}$ : el Acontecimiento de la revelación del Ser -del horizonte del significado que determi na cómo percibimos y nos relacionamos con la realidad- en el pensamiento de Heidegger; y, en el Big Bang (o simetría rota), el Acontecimiento primordial a partir del cual surgió nuestro universo entero, en el enfoque óntico, sostenido por la cosmología cuántica (2014, p. 18).

2 Vale la pena aclarar que estos son los únicos Acontecimientos que son nombrados con mayúscula en el texto, ya que en la actualidad estos son los grandes "Acontecimientos" sobre los cuales se encuentra la discusión central del problema. Por ello, los otros acontecimientos no alcanzan esta relevancia y se escriben intencionalmente con minúscula. 
De este modo, el autor asume al "acontecimiento como un efecto que excede a sus causas", ya que "la característica fundamental de un acontecimiento: [es] la aparición inesperada de algo nuevo que debilita cualquier diseño estable" (2014, p. 15), reafirmando así, que el fenómeno se relaciona con el cambio del entorno, de ahí que se deba asumir como "una transformación devastadora de la realidad misma” (2014, p. 18).

En síntesis, el acontecimiento es una experiencia de ruptura, producto de la expresión de una fuerza que excede las posibilidades de control del ser humano, lo cual alude a una experiencia dolorosa, ya que pone en evidencia el finito poder de las personas y su duración limitada. De ahí que se pueda sostener que el fenómeno haga referencia a la manifestación de lo real, que echa abajo o redefine cualquier realidad. "Acontecimiento como imposición -como cambio en nuestra relación con la realidad- al acontecimiento como un cambio radical de la realidad en sí misma” (Žižek, 2014, p 40). Empero, ¿cómo media este fenómeno el ser humano? Para responder a la pregunta es necesario pensar el concepto en relación a la psiquis, es decir, como el ser humano logra mediar lo real por medio de lo simbólico y lo imaginario.

\section{Los tres acontecimientos del psicoanálisis (Waterfall by Escher)}

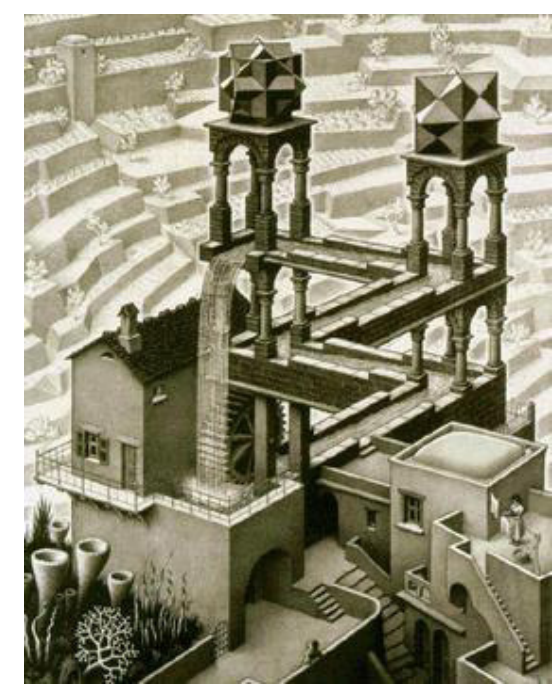

La Cascada (Waterfall) (1961) M.C Escher. Litografía. 
Parece adecuado empezar el apartado con el ejemplo que el mismo Žižek plantea, pero que no desarrolla, esto es, la interpretación de la litografía La cascada, de Escher (1961), como símil que puede ilustrar cómo sucede el fenómeno del acontecimiento en la psiquis. Pero, ¿qué tiene que ver esta obra de arte con los tres niveles del acontecimiento del psicoanálisis? Al igual que en la estampa de Escher, los tres niveles del psicoanálisis son una conexión que hace un solo suceso, ya que estos se entrelazan para componer una sola imagen o acontecimiento, pero, como representa la obra, si hace falta alguna de las partes, la realidad del ser humano no sería posible como tampoco ocurriría la caída del agua en La cascada.

Por tanto, es la conexión entre los tres niveles, la que permite que $L a$ cascada (psiquis humana) acontezca. Sin embargo, es evidente que esta explicación no es suficiente, en razón de que aún no es claro cuáles son los tres niveles del acontecimiento del psicoanálisis. Para entrar en materia, Žižek presenta la obra de W. Shakespeare Sueño de una noche de verano en el acto V: I (diálogo de Teseo). A partir de ahí, se pregunta por cómo el loco, el amante y el poeta se relacionan con la realidad, dando a entender que existe una posible relación entre las "tres dimensiones fundamentales del ser humano" que propuso Lacan en el siglo anterior: "Lo imaginario", "Lo real" y "Lo simbólico" (los tres niveles de la cascada de Escher).

De esta manera, se hace posible establecer la siguiente relación: "el loco" como "lo imaginario", "el que confunde un objeto real con otra cosa" (2014, p. 107); "el amante" como "lo simbólico" "conserva la realidad del objeto amado, que no se invalida, sino que solamente se «transustancia» en la apariencia de una dimensión de lo sublime" (2014, p. 107); y "el poeta" como "lo real", "la trascendencia queda reducida a cero, es decir, la realidad empírica se "transustancia»- no en una expresión/materialización de nada" (2014, p. 107). Žižek desarrolla su postulado así:

quizá podamos utilizar esta triada shakesperiana del lunático, el amante y el poeta como herramienta para proponer una clasificación de acontecimientos basados en la triada lacaniana de lo imaginario, lo simbólico y lo real: un lunático habita en la dimensión imaginaria, confundiendo la realidad con la

3 Conviene recordar que también Platón en la República (580d), había establecido "tres dimensiones fundamentales del ser humano", a saber, la distinción entre alma concupiscible, irascible y racional. 
imaginación; un amante identifica a la persona amada con la cosa absoluta en un cortocircuito simbólico entre significado y significante que sin embargo mantiene el hueco que los separará para siempre (el amante muy bien que en realidad, su amado o amada es una persona corriente, con todos sus fallos y debilidades): un poeta hace que un fenómeno surja contra el fondo del vacío de lo real (Žižek, 2014, p. 107).

Pero, ¿basta con esta relación para entender el acontecimiento del psicoanálisis? De seguro que no. Por ello, Žižek propone lo que significa cada una de las tres dimensiones para Lacan, exponiendo, en primera medida, que lo imaginario tendría que ver con el dominio de la apariencia y de cómo aparecen las cosas para el ser humano. Esto quiere decir que este nivel es la experiencia vivida de la realidad, de los sueños y las pesadillas.

En segunda estancia, está la dimensión simbólica, o lo que llama Lacan: "el gran otro", lo cual es el orden invisible que estructura nuestra experiencia de la realidad, a partir de normas y significados que condicionan las maneras de cómo y qué vemos. Y, por último, está lo real, lo cual, para J. Lacan, es más que una realidad externa, puesto que esta tiene que ver con lo imposible que no puede ser directamente ni experimentado o simbolizado.

Esto último es lo que Žižek plantea (2014, p. 108) como "el encuentro traumático de extrema violencia que desestabiliza nuestro universo entero de significado. Como tal, lo real solo puede discernirse en sus huellas, efectos y consecuencias". Siguiendo este orden de ideas, nos proponemos adentrarnos en el fenómeno de lo real en la realidad humana, siendo, entonces, nuestro primer nivel en donde buscaremos comprender ¿qué sucede cuando el ser humano se enfrenta a la cosa Divina?

\section{Lo real: enfrentándose a la cosa divina}

¿Cómo se puede entender la experiencia psicológica como acontecimiento? Slavoj Žižek (2014, p. 19), plantea que la posibilidad de "la experiencia del Yo como un suceso puramente acontecimental; [se encuentra en] la inmanencia de la ilusión de la verdad que hace que la misma verdad sea acontecimental; un trauma que desestabiliza el orden simbólico que habitamos; el surgimiento de un nuevo «significante maestro», un significante que estructura todo el significado". Lo anterior, en líneas generales, nos obliga a entrar en la 
reflexión de cómo se estructura cada nivel en el psicoanálisis. Para entender lo expuesto, empezaremos por ocuparnos de la experiencia del Yo frente a lo real, es decir, tenemos que reflexionar sobre el suceso de enfrentarse a la cosa de lo real (la verdad).

Para Žižek, este primer aspecto se relaciona con "el encuentro traumático con una cosa divina [la cual] es el acontecimiento como lo real” (2014, p. 110). ¿Qué significa esto? Siguiendo el texto se entiende que la irrupción de lo real en la realidad está sustentada en el cambio de significaciones en la experiencia de la existencia del ser humano, puesto que cuando lo real irrumpe, genera una ruptura y modifica todo el orden simbólico del mundo.

Asunto que nos lleva a la siguiente pregunta: si lo real es lo que cambia el orden de la realidad, ¿es posible que el significante devenga de lo real? Para responder a lo anterior, se plantea una salida parcial, la cual solo sería posible si se asume esa experiencia como una interpretación que se genera entre el símbolo y lo que se manifiesta de la cosa divina, es decir, lo que está más allá de la realidad. Pero, si lo real es algo inalcanzable ¿cómo podemos acercarnos a su acontecimiento en tanto encuentro traumático con la cosa divina?

Lo fundamental en relación con el cuestionamiento anterior, según Žižek, es entender cómo se llega al enfrentamiento con lo real a través de la experiencia del significante maestro, en donde es necesario tomar una distancia de la cosa divina, por dos motivos. Primero, por la limitación de definición que el ser humano posee frente a las cuestiones del campo de lo real (o del ser según el caso).

Y, segundo, porque si el ser humano no interviene la manifestación de lo real por medio del símbolo, se enfrenta cara a cara con una locura despiadada de lo que lo excede, ya que el acontecimiento traumático de afrontar a la cosa divina ${ }^{4}$ (como, por ejemplo, vivenciar el apocalipsis cristiano) es una

4 Este aspecto de mediar el enfrentamiento a la cosa divina por medio del símbolo, nos permite inferir que, la representación de lo Divino, no es otra más que la reducción de su nefasto efecto para el hombre, ya que, si esta experiencia se diera de manera directa, lo único que podría experimentar este es su entera destrucción. En relación con esta idea, Umberto Eco (2007) en la Historia de la fealdad, plantea que el apocalipsis del apóstol Juan, es la representación de la ira sagrada de la cercanía de Dios a la tierra (el fin de la historia a partir de la segunda venida de Cristo al mundo). Según Eco, citando al apóstol Juan (2007, p. 74) dice "antes de que se abra el séptimo sello y siete ángeles que están de pie ante Dios se preparan para tocar sus siete trompetas. Y cada toque de una de las trompetas caen sobre la tierra granizo y fuego y la abrazan, la tercera parte del mar se convierte en sangre, mueren todas las criaturas, caen estrellas y se reducen en tercio los planetas; se abre el pozo del abismo, y de él salen humo y langostas”, a lo que continua diciendo en la página siguiente que, 
experiencia que debe estar mediada por el significante maestro, el cual permite proximidad segura con el horror de estar frente a lo que excede al ser humano.

De esta manera, se resalta parcialmente que el acontecimiento del psicoanálisis de Žižek, se asume como la mediación que hace el significante entre la manifestación de la cosa divina y el ser humano. Es decir, lo simbólico regula la intervención de lo real, lo normaliza y lo acerca a una experiencia en la cual se reducen los efectos devastadores en el mundo.

En otros términos, si el ser humano no mediara lo real por medio del significante, no existiría la realidad, porque sería la manifestación total de lo real (a manera de ejemplo hablaríamos en el caso cristiano del más allá o de la eternidad). En concordancia, se deduce que el acontecimiento traumático por excelencia es acercarse sin mediaciones a la cosa divina, "el auténtico horror no sucede cuando Dios nos abandona, sino cuando Dios se acerca demasiado a nosotros" (Žižek, 2014, p. 111).

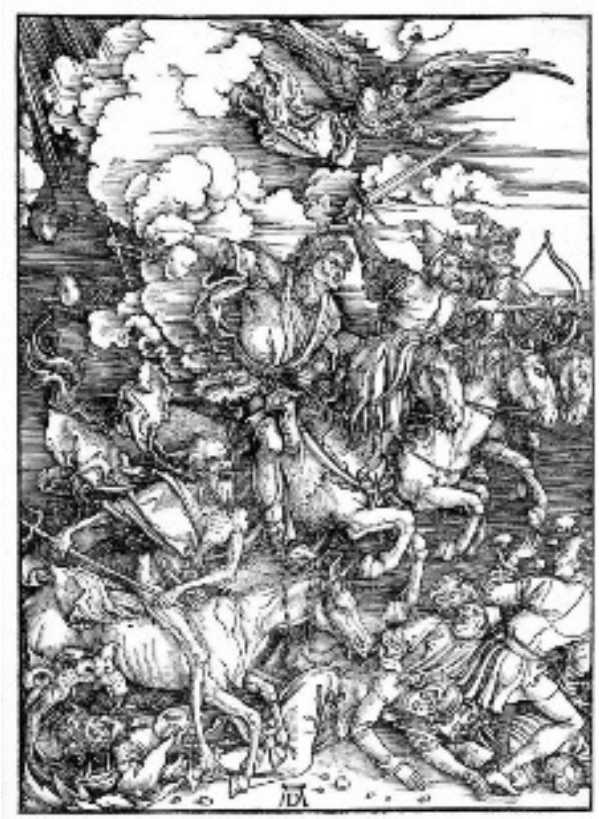

Los cuatro jinetes del apocalipsis (1511) Alberto Durero.

esta intervención simbólica tiene unos efectos que a su vez logran configurar la realidad, ya que "la influencia históricamente más visible del texto de Juan fue en cualquier caso de carácter social y político y tiene que ver con los «terrores del milenio» (2007, p. 78)" lo cual, a nuestro parecer fue la propagación de la angustia de la cercanía con Dios, sobre todo en el medioevo. 
En síntesis, esta experiencia sería el primer nivel del acontecimiento del psicoanálisis, cuando lo real se deposita en lo simbólico. Con todo, ¿cómo interviene lo simbólico en el acontecimiento del psicoanálisis? El anterior asunto nos lleva a pensar en el concepto de significante maestro, siguiente nivel de la triada lacaniana, planteada por Žižek y analizada desde la obra de Escher.

\section{Lo simbólico: la nueva armonía que se relaciona con el pasado perfecto}

Slavoj Žižek llama la atención sobre un sonido de tambor que retumba en este nivel, su intérprete es el poeta Arthur Rimbaud 5 , el cual, con esta metáfora, advierte que el golpe sobre el instrumento es el que descarga el sonido y este es el que inicia la armonía. Ésta es para Žižek, "el significante maestro (...) este momento acontecimental es el momento en el que el significante una forma física que representa el significado- cae dentro de lo que significa, dentro, quiere decir, en que el significado se convierte en parte del objeto que designa” (2014, p. 121). Dicho con otras palabras, para este filosofo el significante maestro es un pacto social, un acuerdo sobre el cual se construye el terreno común de la cultura:

...el pacto social, la unidad que este significante impondrá no será solo ilusoria, es decir, no será únicamente una máscara imaginaria que cubra las diferencias que continúan existiendo. En cuanto la imposición de este significante sirve como punto focal para un movimiento político real que acabe por tomar el poder, establece su propia realidad social: la gente efectivamente colabora, incluso si a todos les parece que lo hacen por su propio beneficio. No importa si algunos grupos utilizan este significante con cinismo, lo que importa es que participan en el espacio socio-simbólico bajo este estandarte.

Ahora bien, si el significante maestro es un pacto que se impone y toma el control de la realidad, este puede ser visto como un punto de encuentro

5 "Un golpe de tu dedo sobre el tambor descarga todos los sonidos- e inicia la nueva armonía. - un paso tuyo es el levantamiento de los nuevos hombres y su- marcha. - vuelves la cabeza: ¡el nuevo amor j- Giras la cabeza, ¡el nuevo amor $\mathrm{i}$ - "cambia nuestros destinos, acribilla las plagas, empezando- por el tiempo", te cantan los niños, "levanta de donde puedas-las sustancias de nuestras fortunas y de nuestros deseos»-te ruegan. - llegada desde siempre, te marcharás a todas partes”. (Arthur Rimbaud, 1873) Á une raison, tomado el 28/6/2018, de https:// www.poetryfoundation.org/poets/arthur-rimbaud 
entre la realidad y lo real, que se deposita en un significado que se establece como lo común. Por ello, el cambio de significación social o su alteración logra condicionar las formas de representación en la sociedad. Al punto de generar nuevos rasgos visibles de lo que no se puede comprender como cultura, porque media el enfrentamiento con la fuerza de la cosa divina que impone o devela sentido y, a su vez, interviene en la designación simbólica del mundo (lo imaginario), porque modifica la perspectiva de la existencia.

Lo anterior permite entender que lo significante sucede al depositar en el significado social una relación interpretativa entre la cosa divina, el símbolo y lo que se piensa que son dichos aspectos. Así, el significante maestro es aquello que se reconstruye constantemente en el mundo, según su multiplicidad de la cultura. Es decir, este es una interpretación social que transforma la concepción de la existencia colectiva. Es así como, se entiende que este significante, se trata de un acontecimiento de condición mutante ${ }^{6}$ que, a nuestro entender, no se deja de relacionar con las manifestaciones variables del ser en las épocas, en correspondencia con las interpretaciones éticas, políticas y estéticas de los seres humanos.

Ahora bien, se podría contemplar al suceso del significante maestro, como un cambio en las nociones de verdad en referencia a lo social, lo cual, parafraseando a Deleuze (2002), en Diferencia y repetición, sería una situación en la que no se trata solo de introducir un orden en los conceptos, sino que, por el contrario, la significación implicaría necesariamente, una diferenciación en la cual se establezcan estatus entre los conceptos que fundan y los fundados, ya que estos últimos dependen de la necesidad que garantizan los primeros (creadores de sentido).

En relación con lo anterior, afirma François Zourabichvili (2004, p. 24), en Deleuze, una filosofía del acontecimiento, que "mientras el comienzo sea pensado como fundamento, estará sometido a un reconocimiento inicial que toma su forma en el sentido común”, o sea, que es una arbitrariedad que un

6 A nuestro modo de ver, esta perspectiva simbólica de Žižek, en el significante maestro, es deudora del pensamiento acontecimental de Gilles Deleuze, por varios aspectos: primero, porque es una noción no historicista (gran diferencia del francés con el pensamiento de Hegel, aunque no se puede negar que este a su vez tiene puntos de encuentro). Segundo, porque prima el símbolo y su significado, como búsqueda que obedece a la multiplicidad de sentidos de la verdad. Y tercero, porque, como resalta, François Zourabichvili (2004, p. 11), el filósofo francés siempre estuvo dispuesto a la meditación de "una filosofía extraña. Constantemente innovadora y meticulosamente terca, estacionaria y mutante (...) un pensamiento ocupado siempre de un elemento variable, inseparablemente ético, estético y político". 
significado social se edifique por sí mismo. Este autor advierte que esto no es absolutamente inmutable, o que no garantiza que no pueda llegar a suceder, ya que "solo tenemos ante la vista su pasado, eminentemente contingente, pasado que no podría valer por un centro o por referencia absoluta”. Lo anterior, aunque problemático, es muy lógico, ya que siguiendo el pensamiento de Deleuze ${ }^{7}$, la verdad no es un eterno absoluto, esta debe ser sometida en perspectiva de multiplicidad.

Se puede asumir entonces al acontecimiento del significante maestro como: el cambio de los conceptos de verdad en referencia a lo social, en donde no solo se permea el presente y el futuro, sino que se logra modificar la percepción del pasado. Lo anterior es lo que Gilles Deleuze llama una relación acontecimental con el "pasado puro" ${ }^{8}$, en tanto que logra una transformación del presente, que consigue cambiar la percepción del pasado, así como el direccionamiento hacia el futuro.

Lo expuesto permite decir que, sin duda, el significante maestro es un pacto susceptible de cambio, cuando la interpretación de lo social se modifica o se imagina de forma distinta, haciendo de este modo que se reestructure desde su pasado hasta su futuro, poniendo en evidencia que el tiempo remoto no es, tan solo, un inmodificable, como tampoco lo es el presente o el futuro, puesto que, el tiempo es vulnerable al cambio y a la interpretación.

Sin embargo, Žižek advierte que el significante maestro, por su condición de orden simbólico, inconscientemente al ser reconocido y aceptado, produce en los seres humanos una sensación de permanencia absoluta, es

7 ESPERÓN, J. (2014) en El acontecimiento y la diferencia en la filosofía de Gilles Deleuze, propone que la teoría acontecimental de Deleuze, es deudora de Friedrich Nietzsche en lo concerniente a que sus reflexiones a partir de "El eterno retorno" y la «voluntad de poder». Lo anterior, no es un aspecto superficial, ya que estas herramientas le ayudan al pensador francés a constituir una perspectiva, en donde el acontecimiento es una interpretación dada desde diversos flujos divergentes en distintos tipos de cuerpos, en los cuales se generan diversos sentidos que devienen de variadas voluntades. Es decir, que el acontecimiento, no es la manifestación de la Verdad (una sola absoluta o ideal), por el contrario, en él, convergen las múltiples verdades. Ahora bien, para este autor, la perspectiva deleuziana, puede ser entendido, no como un devenir histórico (ni en el espíritu de la totalidad de la historia o en la manifestación del ser de una era) sino "como una singularidad sin modelos o esencia propuesta; pues la originalidad de todo acontecimiento resulta ser el movimiento a partir del cual lo nuevo emerge, lo no previsto irrumpe en la realidad y escapa de los límites de la historiografía, porque en todo acontecimiento hay un resto no histórico, inaprensible que excede los límites del discurso"

8 Frente a este asunto Žižek piensa el acontecimiento del significante maestro, desde la perspectiva deleuziana del pasado puro y para explicarlo, cita a T. S. Eliot (1921), Traditional and the individual talent a partir del cual resalta lo siguiente: "el orden existente está completo antes de que llegue una nueva obra; para que el orden persista después de que la novedad sobrevenga, todo el orden existente debe ser, aunque ligeramente, alterado; y así, las relaciones, las proposiciones, los valores de cada obra de arte hacia el todo se reajustan” (Žižek, 2014, p. 124). 
decir, pareciera que estuviese desde siempre, aun cuando no existía ${ }^{9}$. Pero, ¿de dónde emerge esta nueva perspectiva que logra modificar los significantes maestros al punto que logran consolidar unos nuevos? ¿Cuál es el ingrediente que coadyuva a que estos pactos sociales de la realidad se modifiquen? Sin duda, los cuestionamientos anteriores nos permiten pensar en lo imaginario, tercer nivel de la cascada de Escher, el cual es la conexión de lo simbólico a partir de los sentidos.

\section{Lo imaginario}

Como se había anticipado, el nivel de lo imaginario lleva implícita una carga de emotividad. Es por ello que no se podría aislar de los sentidos, pues existe una sensación que se simboliza en forma de abstracción de lo intangible (lo real), que invade la realidad y sus acuerdos sociales. Es decir, este fenómeno interpreta la experiencia del acercamiento con lo real al depositarlo en lo simbólico. De esta manera, se puede entender como lo que se expresa por medio de símbolos, sensaciones y emociones puede llegar a manipular y afectar la experiencia de los seres humanos con la realidad. Como en el caso del arte, en donde su sentido imaginario, depositado en la obra, tiene la posibilidad de hacer sentir horror sin que este sea en sí una experiencia de vulnerabilidad tangible.

En síntesis, la concepción lacaniana del acontecimiento psicoanalítico, como la cascada de Escher, dependen de que se generen conexiones entre los tres niveles mencionados (real-simbólico-imaginario), es decir, que si alguno de los aspectos nombrados falta, la caída del agua en la litografía como la realidad humana sería imposible. Ahora bien, aún nos falta, para redondear el propósito del presente texto, el cual es dar cuenta de la cuestión traumática del acontecimiento para Žižek

9 Esta condición, para Žižek se podría entender como un efecto retroactivo, el cual hace ver al significante maestro que desaparece como si nunca hubiese existido. Por ello, la transformación del pacto social es un cambio que despoja a su antecesor de toda relevancia, para que de esta manera se logre crear una nueva realidad, que establece a la existencia humana una nueva narrativa. 


\section{Sobre el acontecimiento traumático (a manera de conclusión).}

En líneas generales, y retomando algunas ideas desarrolladas en el presente texto, el concepto acontecimiento es una manifestación de un suceso que genera un cambio en el mundo. Pero también puede ser una referencia, que da a entender que se está experimentando un suceso que no es cualquier hecho, siendo así entonces algo que ocurre y que está por fuera de lo común.

De la misma manera, se puede pensar el acontecimiento como una experiencia que mueve las fibras más profundas del ser humano, que logra provocar en él cierta reflexión frente a la existencia. Que es una sensación de gran intensidad y que su manifestación en el mundo está cercana al misterio, a una fuerza que estremece, a algo que está dentro y fuera de la razón. Es decir, el acontecimiento tiene que ver con una vivencia en el mundo que genera una sensación de inestabilidad, contingencia o incluso de transformación. A pesar de esto, dichas definiciones como es sabido solo sirven de acercamiento.

Pero, ¿qué es un acontecimiento? Sin duda, la pregunta remite a múltiples posibilidades. Sin embargo, como señala Žižek (2014, p. 78.) este es: "una ruptura con el pasado: nada siguió igual después de que entraran en escena”, lo cual en el caso de la experiencia humana, se asume como "algo traumático, perturbador, que parece suceder de repente y que irrumpe el curso normal de las cosas; algo que surge aparentemente de la nada, sin causas discernibles, una apariencia que tiene como base nada sólido".

Es decir, el acontecimiento es para el ser humano una experiencia "traumática" indiferente, si este se relaciona con problemas de la psiquis, el símbolo, el lenguaje, la política, la realidad, la filosofía, la ciencia, la religión, el arte o la tecnología, porque el acontecimiento es una experiencia en donde se da cuenta que se está modificando el mundo y la realidad, pero que frente a ello lo único que puede hacer es acogerse a su cambio sin ningún tipo de resistencia.

Otro tópico de la perspectiva traumática de S. Žižek es que el acontecimiento marca al mundo de manera contundente, al punto de generar un antes y un después. En otras palabras, el asunto traumático de un acontecimiento para el ser humano, se puede interpretar como la misma experiencia de existir, la cual se agudiza traumáticamente cuando la vida es sometida a algún tipo de 
cambio. Es por esto que el fenómeno se asume como ruptura de la realidad que genera traumas al modificar parte o la totalidad de la existencia.

Lo expuesto hasta aquí lleva a pensar un aspecto fundamental de la noción traumática del acontecimiento, a saber, que esta vivencia de ruptura es producto de la expresión de una fuerza que excede las posibilidades de control del ser humano, lo cual alude a una experiencia dolorosa, ya que pone en evidencia lo finito del poder de las personas y su duración limitada. Ahora bien, no se puede omitir que el concepto refiere por sí mismo a un suceso de una anormalidad extraordinaria. Expresado de otro modo, "un acontecimiento es por consiguiente el efecto que parece exceder sus causas- y el espacio de un acontecimiento es el que abre por el hueco que separa un efecto de sus causas" (2014, p. 17).

Ahora bien, recordemos que el acontecimiento no es solo un cambio intrascendente, sino el suceso que redefine o destruye el planteamiento de cómo nos relacionamos con lo real. Es evidente, entonces, que el fenómeno tiene una consecuencia traumática sobre el ser humano, la cual es marcada por el poder de lo real ${ }^{10}$ que le condiciona la existencia. Lo anterior cobra sentido al recordar que lo real, para Slavoj Žižek, es "el encuentro traumático de extrema violencia que desestabiliza nuestro universo entero de significado. Como tal, lo real solo puede discernirse en sus huellas, efectos y consecuencias" (2014, p. 108).

En tal sentido, "son los acontecimientos los encargados de cambiar los paradigmas sobre los que se cimienta una época y, por esta razón, intervienen en la velocidad con las que se efectúan las transformaciones de nuestro entorno"11. Este aspecto de lo real, que se hace manifiesto en el acontecimiento, implica entonces que la existencia del hombre está más allá de lo humano, pero tiene que ver con lo humano.

\footnotetext{
10Lo enunciado conduce a pensar en un aspecto sustancial del acontecimiento, a saber, que este tiene que ver con la verdad que se hace manifiesta en lo real. Situación que además de abstracta para el ser humano (porque lo excede en sus posibilidades), es sumamente traumática porque pone en evidencia, como ya se había señalado, que las fuerzas que intervienen en el mundo de manera contundente no están del todo en el dominio de las personas. Ahí radica el problema de ambigüedad del concepto, por ello, durante la historia de la filosofía lo real ha sido nombrado de diversas maneras, ejemplo de ello, es el asunto de la "Cosa Divina" para ПiПek, las "Ideas" para Platón o Descartes, el "ser" para Heidegger y lo que se deposita en la "Razón”. Desde esta óptica, se pone en evidencia que, la realidad humana no es más que la experiencia de la subjetividad eterna de estar en el mundo entre el acierto y el error.

11 Reyes, B. (2015). Libros. ŽIŽEK, Slavoj (2014). Acontecimiento. Madrid: Sexto piso. Universitas Philosophica 65, año 32. Julio- diciembre. ISBN: 0129-5323
} 


\section{Referencias}

Deleuze. Gilles. (2002). Diferencia y repetición. Amorrortu editores: Buenos Aires, Argentina. Eco, Umberto. (2013). El nombre de la rosa. Editorial Deboslsillo: Bogotá, Colombia.

Eco, Umberto. (2007). La historia de la fealdad. Editorial Lumen: Torinese, Italia.

Esperón, Juan. (2014). El acontecimiento y la diferencia en la filosofía de Gilles Deleuze. publicado en la revista filosófica Nuevo Pensamiento.

Reyes, Bryan. (2015). Libros. Žižek Slavoj. Acontecimiento. Madrid: Sexto piso. Universitas Philosophica 65, año 32. Julio- diciembre. ISBN: 0129-5323.

Žižek, Slavoj. (2014). Acontecimiento. Editorial ensayo sexto piso: Madrid, España.

Zourabichvili, François. (2004), Deleuze. Una filosofía del acontecimiento. Amorrortu editores: España. 